\title{
Energy and macronutrient intakes in the Diet and Nutrition Survey of Infants and Young Children 2011 (DNSIYC)
}

\author{
A. M. Stephen ${ }^{1}$, J. P. Sommerville ${ }^{1}$, H. Henderson ${ }^{2}$, D. A. Pell ${ }^{1}$, R. E. Allen ${ }^{3}$ \\ ${ }^{1}$ MRC Human Nutrition Research, Cambridge, UK, ${ }^{2}$ Nat Cen Social Research, London, UK and ${ }^{3}$ Nutrition Branch, \\ Department of Health, London, UK
}

The National Diet and Nutrition Survey assesses the dietary intakes and nutritional status of a representative sample of the population of England, Scotland, Wales and Northern Ireland, aged $\geqslant 18$ months ${ }^{(1)}$. Recognising the need for similar information about those $<18$ months, the Department of Health commissioned a survey of infants and young children 4-18 months, which was carried out in 2011. Intakes of energy and macronutrients are reported here.

The sample was drawn using a multi-stage random probability design from Child Benefit records (at the time a universal credit with a take up rate of around 98\%), in two waves to ensure sufficient numbers at each end of the age range; those aged 4 and 17 months were prioritised. Background information was collected using home interview for details of family dietary habits, socio-demographic status and health information, feeding practices, and eating patterns. Dietary data were collected using an estimated food diary of four consecutive days. Two versions of the diary were developed, one for 4-8 months and one for 9-18 months. Diaries were coded using DINO (Diet In Nutrients Out), HNR's dietary recording and analysis system, also used in NDNS. Food composition was from the Department of Health's Nutrient Databank (Year 3 of NDNS 2010/11). Completion of at least three diary days was considered fully productive. Results were subdivided into 4 age groups, 4-6 months, 7-9 months, 10-11 months, and 12-18 months. Weighting factors were applied to ensure representativeness ${ }^{(2)}$.

There were 2,683 fully productive children in DNSIYC, a response rate of $62 \%$, with the achieved sample very close to the UK population in terms of age, sex, ethnicity and region. Mean daily energy intake from all sources for children 4-6 months was 2.93 MJ (696 kcal), $3.31 \mathrm{MJ}$ (788 kcal) for 7-9 months, 3.61 MJ (859 kcal) for 10-11 months and $4.07 \mathrm{MJ}$ (967 kcal) for 12-18 months. Overall, $75 \%$ of boys and $76 \%$ of girls exceeded the EAR for energy, with increases with age: 59\% and 52\% for 4-6 months, up to $88 \%$ for 12-18 months. Mean protein intakes were well above the RNI in all age groups, providing $10 \%$ of energy for 4-6 months increasing to $16 \%$ for $12-18$ months. Total fat provided $41 \%$ energy for $4-6$ months, $36 \%$ for $7-9$ months and $35 \%$ for $10-18$ months. Saturated fatty acids provided $15-18 \%$ energy across all age groups, contributions decreasing with age. Total sugars provided $38 \%$ energy for children 4-6 months, 33\% 7-9 months, $29 \%$ for 10-11 months and 26\% for 12-18 months. Non-milk extrinsic sugars intake increased with age, from $4 \%$ energy for $4-6$ months to $8 \%$ for $12-18$ months.

Results indicate that overall, UK infants and young children are eating sufficient energy and an appropriate balance of macronutrients. Energy intakes increased along with the \% of children above the EAR with age following the introduction of complementary foods. Examination of food contributions may shed light on the sources of this additional energy.

1. Department of Health. National Diet and Nutrition Survey http://transparency.dh.gov.uk/2012/07/25/ndns-3-years-report/.

2. Department of Health. Diet and Nutrition Survey of Infants and Young Children. http://transparency.dh.gov.uk/2013/03/13/dnsiyc-2011/. 